821.163.6-31.091Levstik V.:821.131.1-31.091Balzac H.

\title{
CONTACTS LITTÉRAIRES FRANCO-SLOVÈNES DURANT L'ENTRE- DEUX-GUERRES. HILARIJ PERNAT DE VLADIMIR LEVSTIK ET LE COUSIN PONS DE BALZAC
}

\author{
Florence Gacoin-Marks
}

\begin{abstract}
Résumé
La présente contribution a pour objectif de montrer en quoi le roman Hilarij Pernat de Vladimir Levstik, à la fois écrivain slovène et grand traducteur de la prose française du XIX ${ }^{\mathrm{e}}$ siècle, semble avoir été largement inspiré par Le Cousin Pons de Balzac. Après avoir dégagé les éléments thématiques (au sens large) rapprochant les deux œuvres (structure de l'intrigue, thèmes et motifs, personnages, ...), il convient de mettre en évidence ce que Vladimir Levstik a retenu de l'esthétique balzacienne dans sa propre esthétique (discours des personnages, incipit/clôture du récit, veine satirique ...).
\end{abstract}

Mots clefs: contacts littéraires, Vladimir Levstik, Honoré Balzac, réalisme, veine satirique, études de réception

Si l'hypothèse d'une influence esthétique et technique de Balzac sur la genèse de La Comète bleue de Vladimir Levstik, peut paraître un peu spéculative, ${ }^{68}$ l'influence exercée par le romancier français sur Hilarij Pernat, roman paru quelques années plus tard, est plus immédiatement visible du fait qu'il s'agit, du moins en partie, d'une influence thématique exercée par une œuvre clairement identifiée sur une autre et non uniquement d'une influence diffuse, plus difficilement identifiable.

Hilarij Pernat est paru pour la première fois en 1926-1927, en feuilleton dans la revue littéraire Ljubljanski zvon. Comme il n'a été publié pour la première fois en volume qu'en 1966, nous ne disposons d'aucun document critique susceptible d'éclairer sa réception par le public slovène durant l'entre-deux-guerres. L'édition de 1966 a passé presque inaperçu. De même, aucun chercheur en littérature slovène ou comparée ne s'est intéressé sérieusement à cette œuvre. ${ }^{69}$

Avant d'analyser les similitudes entre les romans français et slovène, il convient de s'interroger sur la connaissance que Vladimir Levstik, futur traducteur d'Illusions

\footnotetext{
${ }^{68}$ Nous avons développée cette hypothèse dans un précédent article paru en slovène en 2005 dans la revue Primerjalna književnost.

${ }^{69} \mathrm{La}$ bibliographie antérieure à nos travaux est donc uniquement composée d'une étude historique éclairant le cadre dans lequel s'inscrit le roman (Grdina 1995).
} 
perdues, de Splendeur et misère des courtisanes et des Paysans, avait de Balzac avant la Seconde Guerre mondiale. Bien qu'il nous soit impossible d'affirmer que l'écrivain slovène avait lu Le Cousin Pons avant 1926, nous pouvons tout de même affirmer que cette hypothèse est plausible et qu'il avait sans aucun doute lu plusieurs romans de Balzac. C'est du moins ce que semble affirmer Albert André Algoud, grand ami français de Vladimir Levstik, qui, relatant leur première rencontre en 1911, nous fournit le renseignement suivant : «Et puis, inhabile à l'éloquence, je dérivai sans transition vers Balzac, et notre entretien prit soudain un tour si enjoué que nous nous fîmes quasiment chasser de la place, la nuit étant venue sans que nous en eussions conscience » (Algoud 1978 : XIV). Ce « rapport de faits » est confirmé par les autres membres de la famille Algoud encore susceptibles d'apporter leur témoignage, ce qui nous amène à conclure que Vladimir Levstik avait bien lu aux moins quelques romans de Balzac avant 1926, et même avant 1911.

\section{UNE HISTOIRE DE COLLECTIONNEUR CÉLIBATAIRE ET D'HÉRITAGE}

Dès la première lecture, la parenté entre Hilarij Pernat et Le Cousin Pons apparaît avant tout dans les similitudes entre l'intrigue et les personnages principaux des deux romans. En revanche, cette impression tend à se dissiper au fil des pages en raison des divergences entre les thèmes secondaires abordés par les deux auteurs.

Dans Hilarij Pernat, Vladimir Levstik propose une histoire très semblable à celle du Cousin Pons, celle d'un sexagénaire célibataire sans héritier direct dont les héritiers effectifs sont des parents éloignés très antipathiques. À partir de cette trame, les deux romanciers développent une intrigue dont les grandes lignes peuvent être considérées comme similaires, même si la fin du roman slovène est radicalement opposée à celle choisie par Balzac.

En dépit de différences significatives, l'histoire développée par les deux romanciers est très proche : le sexagénaire se brouille avec ses héritiers naturels avec qui il n'a aucune affinité véritable, tombe malade et écrit un testament en faveur d'un tiers (la personne de confiance avec qui il vit). Les différences viennent du fait que Balzac a multiplié le nombre des rapaces au chevet de Pons mourant et compliqué l'intrigue autour du testament (le « vrai » testament en faveur de Schmucke est précédé d'un faux testament en faveur de l'État), tandis que Vladimir Levstik a introduit l'existence d'un fils illégitime, véritable héritier naturel de Pernat, et le suspense concernant son retour $\mathrm{du}$ front. Par ailleurs, un fait capital induit un déroulement différent de l'intrigue : dans le roman de Vladimir Levstik, le personnage éponyme est dès le début considéré comme riche, ce qui induit de la part des parents une attitude intéressée dès le début du roman et rend inutiles toutes les intrigues concernant l'évaluation de sa fortune.

De même, contrairement aux apparences, le cadre dans lequel s'inscrit la majeure partie de l'histoire est semblable. Certes, le roman de Balzac se déroule à Paris, mais dans un quartier bien particulier où l'on en vient à oublier que l'on vit dans la capitale : «La rue de Normandie est une de ces rues au milieu desquelles on peut se croire en 
province : l'herbe y fleurit, un passant y fait un événement, et tout le monde s'y connaît » (Balzac 1950: 560). Balzac a donc choisi un cadre étroit, limité, où Pons va se retrouver comme prisonnier des machinations de quelques particuliers. L'histoire d'Hilarij Pernat se déroule dans une petite ville de province (Celje, selon toute vraisemblance), plus particulièrement dans la « rue du jardin » où Blanka Nagerth possède une petite maison resserrée « comme si elle manquait d'espace en haut et sur les côtés » (Levstik 1926: 325). Il s'agit donc, ici aussi, d'un cadre très circonscrit.

On trouve dans Hilarij Pernat certaines scènes qui rappellent Le Cousin Pons.

Dans les premières pages du roman de Balzac et au début du chapitre II de Hilarij Pernat, le lecteur fait la connaissance du personnage éponyme par le moyen d'une scène où le sexagénaire marche dans la rue sous les regards des passants. Chez Balzac, ces derniers se moquent du caractère démodé de son habillement, tandis que, chez Levstik, ils s'étonnent de la rapidité avec laquelle le professeur a vieilli depuis peu. Les deux scènes aboutissent à une biographie du personnage depuis ses origines familiales jusqu'à sa vie au moment de l'histoire centrale du roman.

Plus loin, dans les deux cas, la brouille entre les parents intervient à la suite d'accusations mensongères (Mme Camusot de Marville reproche à Pons d'avoir voulu lui nuire en présentant Brunner à sa famille et Blanka insinue que Pernat défend Stana parce qu'il nourrit à son égard des sentiments coupables); la différence est que, chez Balzac, ce sont les Camusot de Marville qui chassent leur cousin, tandis que c'est le héros de Levstik qui jette sa cousine Blanka dehors.

La chasse à l'héritage est également très semblable. L'atmosphère qui entoure le lit du malade est aussi étouffante chez Levstik que chez Balzac. Dans les deux cas, le malade est pris d'un malaise, qui apparaît à son entourage comme une sorte de « fausse » mort, de mort « ratée » (Balzac 1950: 722-723). En particulier, la scène où Stana surprend les parents en train de provoquer un dangereux courant d'air dans la chambre rappelle plus d'une scène macabre du Cousin Pons. Dans les deux romans, l'une des personnes intéressées par l'héritage va même jusqu'à voler le testament dans le but de le détruire s'il s'avère en sa défaveur; Blanka réussit même là où la Cibot $\mathrm{a}$ échoué, puisqu'elle parvient à brûler le testament de Pernat. Notons que c'est cet acte qui permet de démasquer la Cibot et Blanka.

Enfin, il convient de mentionner les nombreux dialogues entre le personnage principal et son allié (Pons et Schmucke dans le roman français, Pernat et Stana dans le roman slovène) qui ponctuent de la même manière chacun des deux romans.

Il est également intéressant d'examiner la clôture du roman de Levtik. Tandis que le roman de Balzac s'achève par la mort du cousin Pons et de son ami Schmucke (l'un meurt de maladie, l'autre des suites de la machination mise en œuvre contre lui) et sur la vente de la collection, un avenir heureux se dessine pour le personnage slovène et son allié dans le chapitre XVI qui clôt le roman de Vladimir Levstik.

En effet, sans ce dernier chapitre, le roman s'achèverait sur une réplique identique des héritiers naturels assurant le triomphe de l'hypocrisie :

Il dînait trois ou quatre fois par semaine chez moi, reprit-elle, il nous aimait tant ! Nous savions l'apprécier, les artistes aiment ceux qui goûtent leur esprit. Mon mari 
était d'ailleurs son seul parent. Et quand cette succession est arrivée à Monsieur de Marville, qui ne s'y attendait nullement, monsieur le comte a préféré acheter tout en bloc plutôt que de voir vendre cette collection à la criée; et nous aussi nous avons mieux aimé la vendre ainsi, car il est si affreux de voir disperser de belles choses qui avaient tant amusé ce cher cousin (Balzac 1950: 802).

Les deux dames Nákrt, Jean Bellé et son épouse se concertèrent à voix basse ; ensuite, ils déclinèrent unanimement la proposition du docteur. Leur sensibilité et leur amour pour leur bon oncle Hilarius ne leur permettaient pas de l'importuner au milieu des tourments de la mort avec des affaires profanes (Levstik 1927: 590).

Dans les deux cas, les héritiers naturels feignent d'éprouver de la tendresse pour leur parent (le cher cousin, le bon oncle), omettant de rappeler qu'ils se sont fâchés avec lui et ne se sont jamais réconciliés. Mieux encore, ils cachent les vrais raisons de leurs actes (dans les deux cas l'intérêt) sous des airs de compassion : si les de Marville ont vendu toute la collection d'un bloc au comte, ce n'est pas par égards pour leur défunt cousin, mais parce qu'il s'agissait là d'une bonne affaire; de même, les parents de Hilarij Pernat ne souhaitent pas que l'on fasse venir un notaire parce que, en l'absence de tout testament, ce sont eux qui héritent des biens du défunt.

Or, contre toute attente, la situation se renverse : Hilarij Pernat guérit, Milan Kantor rentre de la guerre sain et sauf, retrouvant ainsi son père et Stana qu'il épouse peu après. Par ailleurs, la cousine Blanka se trahit et est jetée dehors par l'oncle en colère... Un vrai conte de fée qui contraste fortement avec la fin sombre et tragique des deux « casse-noisettes » parisiens. Ici, Vladimir Levstik fait preuve d'un grand conservatisme formel. En effet, aux retrouvailles de Hilarij Pernat et son fils Milan Kantor (solution à l'enjeu que constitue le devenir de l'héritage) Vladimir Levstik ajoute deux pages séparées du reste du texte par un astérisque. Cette fin comporte des caractéristiques identiques à celles des clôtures classiques des romans européens antérieurs au naturalisme. Commençant par une phrase exprimant un saut temporel («Six mois avaient passé. »), la fin de Hilarij Pernat propose un bref résumé de ce qu'il est advenu des différents personnages du roman avant de s'achever sur un très conventionnel consentement de mariage.

En réalité, cette fin met en évidence un autre aspect de Vladimir Levstik dont nous avons déjà eu l'occasion de parler concernant La Comète bleue : le sens de la progression dramatique de l'intrigue hérité non seulement d'Alexandre Dumas, mais aussi des grands auteurs dramatiques. L'écrivain slovène est réaliste dans la mesure où il propose dans son œuvre un tableau critique de la société dans laquelle il vit, cependant il n'a pas renoncé à l'intrigue dans le sens classique du terme. La fin de Hilarij Pernat ressemble à la fin d'une comédie de Molière : les « méchants » hypocrites sont démasqués et chassés comme à la fin du Malade imaginaire; quant au héros, il retrouve son fils qu'il marie, cela va sans dire, avec la jeune fille qu'il a élevée comme une fille adoptive. Par cette fin aussi rapide qu'idyllique et caricaturale, Vladimir Levstik termine son étude de mœurs par une note positive croquée en quelques pages. Il convient donc plutôt de considérer cette fin comme une coda optimiste formelle, une sorte de revanche de Pons.

La parenté entre Hilarij Pernat et Le Cousin Pons est également visible dans l'opposition entre deux clans formés, d'un côté, par le personnage titre accom- 
pagné de son allié et, de l'autre, des héritiers soutenus par quelques personnages secondaires.

La parenté entre Pons et Pernat, les personnages éponymes des romans français et slovène, est évidente. Ils sont tous deux sexagénaires et célibataires. Tandis que Pons n'est jamais parvenu à plaire aux femmes, Pernat a opté pour le célibat à la suite d'un amour malheureux..$^{70}$ Amateurs d'art, les deux personnages possèdent une collection qui constitue l'héritage tant convoité. Certes, leurs traits de caractère sont très distincts (le dynamisme et le tempérament sanguin de Pernat contrastent avec le caractère pondéré, presque effacé de Pons), néanmoins il existe un point commun entre eux : la fragilité de leurs nerfs. Tandis que Balzac montre comment Pons tombe malade à la suite d'une brouille familiale, Vladimir Levstik note que Pernat « est un homme aux nerfs plus sensibles que ceux du commun des mortels » (Levstik 1926: 334). Les deux personnages présentent donc un certain déséquilibre émotionnel. Petit détail amusant concernant l'enfance de Pernat : fils d'une harpiste, il était destiné à devenir musicien, mais son père a finalement choisi de faire de lui un professeur " parce qu'il n'avait pas assez de talent pour devenir musicien » (Levstik 1926: 335). En un sens, le personnage slovène est une sorte de Pons raté... Il convient ici de mentionner un autre collectionneur célibataire de la littérature française ayant pu influer sur la genèse de Pernat: Des Esseintes, le héros d'A Rebours de Huysmans. En effet, comme Huysmans, le romancier slovène remonte à plusieurs générations pour expliquer le tempérament de son personnage, constatant que ce dernier « était le descendant de la lignée Pernath dans lequel le vieux sang patricien avait connu sa dernière infidélité par rapport au passé » (Levstik 1926: 334). De manière générale, le passage où Vladimir Levstik revient sur les origines familiales de son personnage présente de fortes similitudes avec la « notice » d'À Rebours.

Pons et Pernat sont tous deux accompagnés d'un personnage que l'on peut - en raison de sa fonction - appeler un adjuvant ou un allié : une personne de confiance avec qui ils vivent. Certes, en apparence, Schmucke et Stana sont très dissemblables, pourtant la fonction de la jeune fille dans l'histoire est très similaire à celle du musicien allemand. Héritière potentielle sans être du même sang que le personnage éponyme, elle est l'objet de toutes les haines. Étant la seule attachée à Pernat par une affection sincère et désintéressée, elle veille ce dernier durant toute sa maladie. Comme Schmucke, elle est amenée à un certain moment de l'histoire à dissimuler au vieil homme un document qui l'aurait affligé (une lettre laissant penser que son fils est décédé), action qui est dévoilée par l'un des personnages cherchant à la discréditer.

Les héritiers naturels de Pernat sont au nombre de trois : Blanka von Nagerth, Jean Bellé et Mimi Plešivec. Autant Jean et Mimi (un profiteur de guerre et une mère de famille, pilier de son foyer) sont des types originaux, autant le personnage de Blanka semble devoir beaucoup à deux personnages du Cousin Pons pourtant très différents : madame Camusot de Marville (la présidente) et la Cibot.

\footnotetext{
${ }^{70}$ La biographie détaillée qu'Anton Šepetavec donne de Vladimir Levstik dans son mémoire de DEA laisse à penser que l'amour malheureux du héros pour une jeune fille qui finit par épouser son meilleur ami est un motif d'origine autobiographique.
} 
Le personnage slovène partage avec la présidente la conviction de ne pas posséder une fortune suffisante non seulement eu égard à ses origines et à son statut social, mais aussi par rapport aux autres membres de sa famille :

Les yeux, encore vifs et caustiques, exprimaient une morgue judiciaire chargée d'une envie contenue. [...] Son caractère, déjà cassant, s'était aigri. Plus vieillie que vieille, elle se faisait âpre et sèche comme une brosse pour obtenir, par la crainte, tout ce que le monde se sentait disposé à lui refuser (Balzac 1950: 551).

Quand $<$ Hilarij Pernat $>$ avait disparu, le visage de Blanka redevenait laid et vieux, plein de tristesse et de déception, plissé dans un masque d'envie inconsolable et de colère inapaisable à l'encontre du monde entier (Levstik 1926: 621).

La colère et l'envie déforment et enlaidissent les deux femmes.

Comme corollaire de cette situation financière, la présidente et Blanka doivent faire face à une autre préoccupation, celle de « caser » au mieux une fille largement en âge de se marier. Mais, tandis que dans le cas de Cécile (âgée de 23 ans) tous les espoirs sont encore permis (à la fin du roman, nous la retrouvons jeune mariée), de son côté Alisa Nagerth est déjà une vieille fille d'une trentaine d'années. Par ailleurs, sa chasse au mari est entravée par un élément extérieur déterminant : la guerre, qui a vidé la ville de sa population masculine.

Une fois dans la course à l'héritage, les intérêts de Blanka, tout comme ceux de madame de Malville, sont défendus également par les notables de la ville. La différence réside dans le fait que les personnages de Balzac agissent par intérêt personnel, tandis que ceux de Levstik suivent à la lettre les us et coutumes ancestraux qui veulent que l'on se range systématiquement du côté des héritiers « légitimes » ou considérés comme tels.

On retrouve également dans le personnage de Blanka Nagerth certaines caractéristiques de la Cibot. En effet, ruinée par son défunt mari, Blanka mène une existence de petite commerçante, de buraliste, qui n'a rien à envier à celle de la Cibot dans sa loge.

Plus tard, le personnage slovène joue dans l'intrigue un rôle identique à celui de la concierge de Balzac : elle assiège le domicile du malade et subtilise le testament, éléments importants dans la mesure où ils contribuent à créer une atmosphère particulière dans les pages du roman relatant la maladie du sexagénaire et la chasse à l'héritage.

Notons que les deux auteurs utilisent pour désigner les personnages se pressant autour de l'héritage un « bestiaire» métaphorique similaire. Chez Balzac, le regard de la Cibot est « un regard de tigre » (Balzac 1950: 619), Fraisier et ses complices sont des « corbeaux flairant leur cadavre » (Balzac 1950: 719) et, pour Schmucke, toute lutte est vaine car « $<\mathrm{c}>$ es homes ond des fizaches de digre » (Balzac 1950: 787); dans le roman slovène, Hilarij Pernat compare ses parents accourant à son chevet à des hyènes (« hijene ») et, effectivement, quelques lignes plus loin le narrateur remarque que le bruit que font ces derniers est « semblable au piétinement d'un fauve s'élançant sans réfléchir après sa proie » (Levstik 1927: 585). Quant à Blanka, « elle attendait dans son bureau de tabac comme une araignée dans sa toile et guettait sur le seuil » (Levstik 1926: 621).

Les similitudes des grandes lignes de l'intrigue, de certaines scènes et des personnages de Hilarij Pernat et du Cousin Pons sont donc suffisamment nombreuses pour 
que l'on puisse songer à un lien génétique entre les deux œuvres. Toutefois, il convient de ne pas négliger la présence d'un autre fil conducteur du roman absent du roman de Balzac : la recherche du fils héritier, qui se développe parallèlement à la chasse à l'héritage. Cette partie de l'intrigue éloigne considérablement le roman slovène non seulement du Cousin Pons, mais également du réalisme en général : tandis que Balzac s'attarde surtout sur la psychologie des différents personnages concourant à la perte des deux musiciens, Vladimir Levstik consacre davantage d'espace aux doutes et tourments qu'endure le personnage principal à la recherche de son fils.

\section{HILARIJ PERNAT ET LA POÉTIQUE BALZACIENNE}

Mais la parenté thématique avec Le Cousin Pons n'est pas le seul lien rattachant Hilarij Pernat au réalisme balzacien. D'autres éléments plus techniques soulignent également l'influence du romancier français sur Vladimir Levstik. Parmi les aspects les plus flagrants, mentionnons l'incipit du roman slovène, la conception du narrateur et surtout l'importance accordée aux particularismes linguistiques des personnages.

Hilarij Pernat commence par un incipit statique descriptif ${ }^{71}$ qui peut être rapproché de celui de nombreux romans de Balzac, en particulier celui du Père Goriot :

À l'angle de la place Mestni trg et de la rue Samostanska, une maison de petite taille, couverte de lichen gris, se serre entre ses voisines, comme si elle manquait de place en haut et sur les côtés. L'entrée sombre donne sur la rue ; le premier étage comporte deux fenêtres exiguës, bordées de vert, donnant sur la place et trois donnant sur la rue ; le rez-de-chaussée avance sur la rue avec deux ouvertures tout aussi tristes dont l'une s'ouvre à l'aide d'un loquet et est décorée de cartes postales jaunies et de journaux datant toujours de plusieurs semaines.

En pleine canicule de l'année de guerre 1917, tous les braves habitants du voisinage admiraient l'aigle impérial qui surmontait cette éminente fenêtre et la planche noire et jaune sur laquelle on pouvait lire l'inscription $» K$. k. Tabaktrafik'. En effet, rares étaient ceux qui déambulaient dans le bourg sans être attirés par cette enseigne; si on n'y trouvait pas de cigares et de cigarettes, on avait au moins l'occasion de faire la connaissance de madame von Nagerth ou Nákrt, selon la prononciation des gens simples, et de sa fille Alisa qui distribuaient les merveilles que proposait le monopole du tabac, la presse patriotique du jour et les nouvelles mondaines récentes dans leur version corrigée et dupliquée (Levstik 1926: 325).

L'écrivain campe un lieu et une atmosphère en décrivant une maison, puis évoque la vie quotidienne dans la ville, ce qui lui donne l'occasion de présenter brièvement l'un des personnages principaux du roman.

Comme dans Višnjeva repatica, le narrateur de Hilarij Pernat est absent de l'histoire mais omniscient. Comme chez Balzac, le récit est caractérisé par une focalisation zéro : l'information fournie au lecteur est complète, de sorte que ce dernier connaît tout, même les pensées les plus intimes des personnages. Les passages focalisés du point de vue de l'un des personnages ne sont pas inexistants, mais sont très rares. Pourtant, dans

\footnotetext{
${ }^{71}$ Nous utilisons ici la terminologie proposée par Andrea Del Lungo dans son article de 1993.
} 
le cadre de cette narration à la troisième personne non focalisée, nous rencontrons la première personne (du pluriel, puis du singulier) semblant vouloir indiquer au début du roman que le narrateur est l'un des habitants de la petite ville où se déroule l'histoire. Ce procédé a pour effet d'installer l'illusion référentielle.

Le Cousin Pons est un roman intéressant également du point de vue linguistique. En effet, dans les passages en style direct, certains personnages parlent un idiome qui leur est propre. Le fort accent allemand de Schmucke et la prononciation chuintante de Rémonencq sont rendus par Balzac à l'aide d'une écriture phonétique (d'une lecture parfois astreignante) ; quant à la langue de la Cibot, elle est proposée au lecteur dans toute sa familiarité, avec ses images pittoresques, ses constructions fautives, son surplus de $« \mathrm{n} »$ :

Soyez tranquille, vous n'avez près de vous n'un bon ami, et, sans me vanter, n'une femme qui vous soignera comme n'une mère soigne son premier enfant. J'ai tiré Cibot d'une maladie que monsieur Poulain l'avait condamné, qu'il lui n'avait jeté, comme on dit, le drap sur le nez, qu'il n'était abandonné comme mort... (Balzac 1950: 619).

Les autres personnages parlent un français standard, exempt de marques régionales ou sociales.

On trouve la même tendance - dans une variante moins appuyée - dans Hilarij Pernat : le personnage éponyme et, dans une moindre mesure, la jeune Stana, parlent un slovène truffé d'expressions françaises et latines, tandis que Blanka von Nagerth et sa fille utilisent un nombre important d'expressions allemandes (non traduites), comme : « Pleurer un fils qui, en comptant sur les doigts de la main, pourrait lui-même constater qu'il n'était pas de lui ... Wie komisch !...» (Levstik 1927: 87). La différence entre l'authenticité linguistique accrue des dialogues dans les deux romans réside dans la signification que revêtent les particularismes des personnages. En effet, chez Levstik, l'opposition linguistique entre le latin et le français d'un côté et l'allemand de l'autre n'a pas uniquement pour but d'accroître l'effet de réel, mais contribue à renforcer l'opposition entre les deux clans en présence et, par ailleurs, revêt une signification idéologique et nationale absente du roman de Balzac. C'est ainsi que les conservateurs pangermanistes s'opposent aux intellectuels partisans d'un état yougoslave indépendant de l'Autriche-Hongrie. Même linguistiquement parlant, Hilarij Pernat et ses proches parents ne partagent pas les mêmes valeurs.

Certains traits stylistiques et narratologiques de Hilarij Pernat marquent donc une parenté réelle entre l'écriture de Balzac et celle de Vladimir Levstik. À ces traits plus aisément saisissables, il convient d'ajouter une tendance générale de l'écrivain slovène à la satire. En effet, même s'il ne porte pas le sous-titre de « roman satirique » comme Višnjeva repatica, Hilarij Pernat relève réellement de la caricature, ce qui, là encore, le rapproche de l'univers romanesque balzacien.

Toutefois, les similitudes étudiées ici ne doivent naturellement pas cacher les éléments qui éloignent Hilarij Pernat du Cousin Pons : du point de vue thématique, l'importance accordée à la lutte de certains Slovènes pour la création d'un état slave et l'absence de ces descriptions et de ces pauses réflexives si caractéristiques de l'écriture 
balzacienne. En dépit de ces divergences, nous pouvons conclure que le roman de Balzac a vraisemblablement joué un rôle essentiel dans la genèse du roman de Vladimir Levstik.

Après la Première Guerre mondiale, le roman français du XIX siècle n'a donc pas fini d'exercer une influence sur le roman slovène. Celle-ci se poursuivra durant les années trente jusqu'au cataclysme de la Seconde Guerre mondiale.

Université de Ljubljana, Slovénie

\section{BIBLIOGRAPHIE}

1.

Balzac, Honoré de. Le Cousin Pons. In : Honoré de Balzac, La Comédie Humaine VI. Paris : Gallimard, zbirka »Bibliothèque de la Pléiade«, 1950 (1847).

Levstik, Vladimir. Hilarij Pernat. Ljubljanski zvon, 1926-1927.

2.

Algoud, Albert André. «Préface ». In : Vladimir Levstik, Gadje gnezdo. Paris : SEDEP, 1978.

Del Lungo, Andrea. « Pour une poétique de l'incipit ». Poétique, 94, 1993, pp. 131-152.

Grdina, Igor. « Vladimir Levstik v času in prostoru ». In : Vladimir Levstik. Hilarij Pernat. Založba obzorja : Maribor, 1995, pp. 209-318.

Šepetavec, Anton. Pisatelj Vladimir Levstik v prvem desetletju 20. stoletja. Magistrska naloga. Ljubljana: Oddelek za slovenistiko, Filozofska fakulteta, 2000. 